\title{
Shape Confirmation of Silver Nanoparticles from Characterization Techniques
}

\author{
Lipi Goswami ${ }^{1}$, Pranayee Datta ${ }^{2}$ \\ ${ }^{1}$ Department of Physics, Girijananada Chowdhury, Institute of Management and Technology, Guwahati, India \\ ${ }^{2}$ Department of Electronics and Communication Technology, Gauhati University, Guwahati, India
}

Email address:

lipigoswami1@gmail.com (L. Goswami)

To cite this article:

Lipi Goswami, Pranayee Datta. Shape Confirmation of Silver Nanoparticles from Characterization Techniques. American Journal of Materials Synthesis and Processing. Vol. 3, No. 2, 2018, pp. 23-29. doi: 10.11648/j.ajmsp.20180302.13

Received: June 10, 2018; Accepted: July 3, 2018; Published: July 31, 2018

\begin{abstract}
The silver nanaoparticles are synthesized via green route using the tea leaves extract and the characterized samples reveal nano formation. The localized surface Plasmon resonance (LSPR) is an important characteristic of metal nanoparticles. From the study of the resonance peak in the UV-Vis spectrum, an interesting fact about the shape of nanoparticles is disclosed. The presence of two peaks in the UV-Vis spectrum gives us a theoretical indication that the shape of the nanoparticles formed is neither spherical nor uniform which exactly matches with the HRTEM pictures of the synthesized nanosamples. The HRTEM picture of the sample where one Plasmon peak is there is characterized mostly by the presence of spherical shape of nps. Moreover, there is distribution of nanoparticles with uniform shape. But the sample with two plasmon peaks in the UV-Vis spectrum is characterized by the formation of nanoparticles with non uniform shape.
\end{abstract}

Keywords: Silver Nanoparticles, Green Route, Localized Surface Plasmon Resonance, HRTEM

\section{Introduction}

Nanoparticles made from certain metals, such as gold and silver, strongly interact with specific wavelengths of light and the unique optical properties of these materials is the foundation for the field of plasmonics [1]. Plasmon oscillation is the phenomena that free electrons in the metal oscillate cooperatively from their equilibrium position where the positive charge of metal bind the ensemble of the free electrons. "Plasmon" means the quasi-particle representation of plasma frequency. The surface plasmon is the plasma oscillations that localize at the surface or interface. Surface Plasmon polaritonsare surface electromagnetic wave that propagates in a direction parallel to the metal/dielectric (or metal/vacuum) interface. Since the wave is on the boundary of the metal and the external medium (air or water for example), these oscillations are very sensitive to any change of this boundary, such as the adsorption of molecules to the metal surface. LSPRs (Localized SPRs) are collective electron charge oscillations in metallic nanoparticles that are excited by light as shown in figure 1. They exhibit enhanced near-field amplitude at the resonance wavelength. This field is highly localized at the nanoparticle and decays rapidly away from the nanoparticle/dieletric interface into the dielectric background, though far-field scattering by the particle is also enhanced by the resonance. Light intensity enhancement is a very important aspect of LSPRs and localization means the LSPR has very high spatial resolution (subwavelength), limited only by the size of nanoparticles [2].

The simplest way to approach the problem is to treat each material as a homogeneous continuum, described by a frequency-dependent relative permittivity between the external medium and the surface. This quantity, hereafter referred to as the materials' "dielectric constant," is complex permittivity. In order for the terms which describe the electronic surface plasmons to exist, the real part of the dielectric constant of the metal must be negative and its magnitude must be greater than that of the dielectric. This condition is met in the IR-visible wavelength region for air/metal and water/metal interfaces (where the real dielectric constant of a metal is negative and that of air or water is positive). Metal nanoclusters have free electrons. They collectively vibrate and interact with phonons. The surface Plasmon resonance (SPR) absorption recorded in spectrophotometer shows a broad band in the UV-spectrum. 
The cluster diameter can be obtained from FWHM $\left(\Delta \mathrm{E}_{1 / 2}\right)$ of the band as [3],

$$
\mathrm{d}=\frac{h v_{F}}{\pi \Delta E_{1 / 2}}
$$

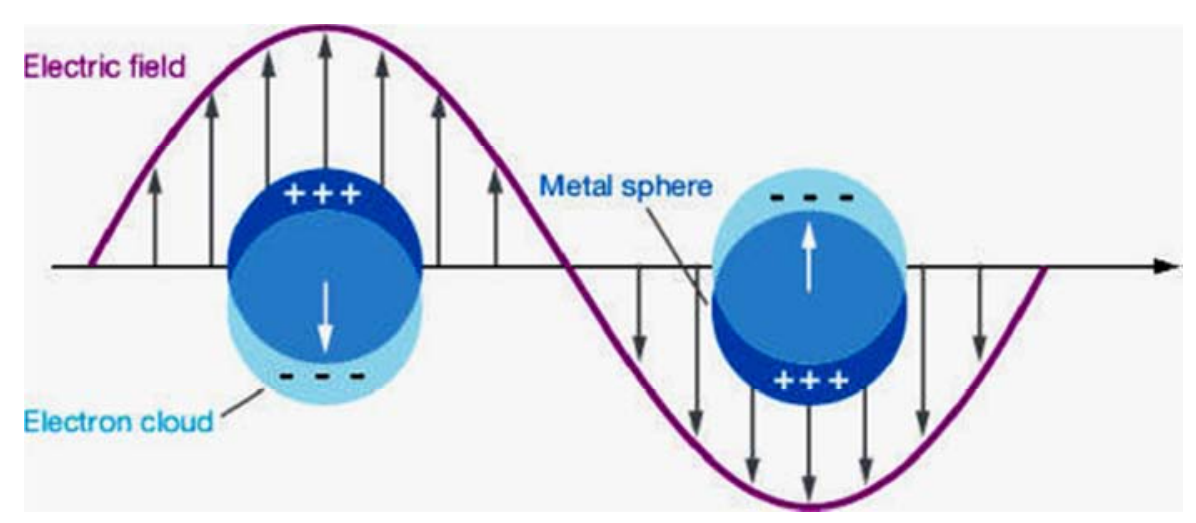

Figure 1. LSPR oscillation (available at http://en.wikipedia.org/wiki/File.

The phenomenon of LSPR, in case of metal nanoparticles is a very important phenomenon which is utilized to study their different behavior for application in different fields. It is seen that metal nanoparticles release heat on exposure to laser beam and when the laser wavelength is in resonance with the surface plasmon resonance wavelength, the nanoparticles release most of the energy in the form of heat [4]. The great enhancement of the local field near the SPR peak could mediate the fluorescence of the materials.

The position and the shape of the absorption spectra depends on particle size and shape, the stabilizer used in the synthesis of the nanoparticles. According to Mie theory, only a single LSPR peak is expected in the absorption spectra of isotropic silver nanoparticles whereas two or more peaks are observed in anisotropic particles [4]. It is found that the number of SPR peaks increases as the symmetry of the nanoparticles decreases. Thus spherical np show one peak and disks, triangular or asymmetric nps show two or more peaks.

The nanoparticles synthesised through chemical route cannot be considered as environment friendly because a lot of chemicals are used which are hazardous forhealth. But recently, the fabrication of nanoparticles through green route has gained interest because the methods are considered safe as they utilise non-toxic chemicals and solvents. Moreover, it does not require the use of any kind of stabilizers. Various environmental friendly materials like plant extracts, bacteria, actinomycetes, fungi and enzymes come under 'green synthesis' arena. The process of using microorganisms for the synthesis of nanoparticles also involves the culture of the microorganisms which is tedious and hence the use of plant or plant extracts is an advantage over the use of microorganisms. These processes can be easily scaled up for the synthesis of nanoparticles without involving any high pressure, energy and temperature conditions.

The following ideal properties in the nanoparticles where $v_{F}$ is the fermi velocity $=1.39 \times 10^{8} \mathrm{~cm} / \mathrm{sec} \Delta E_{1 / 2}=$ Full Width at Half Maximum. prepared by any method are essential [5]

(i). Identical size of all the particles (monosized or with uniform size distribution)

(ii). Identical shape or morphology

(iii). Identical chemical composition

(iv). Identical crystal structure among different particles and within individual particle (the core and the surface of the particle must have the same crystal structure)

(v). Individually dispersed or monodispersed. No agglomeration should be there. If it is there then it must be re-dispersible.

A variety of preparation route by various workers for preparation of $\mathrm{Ag}$ nanoparticles have been reported by Poonam Benjwal [6]. S. Navaladian et al. [7] in the year 2010 reported synthesis of $\mathrm{Ag}$ nanoparticles by thermal decomposition of silver oxalate in water and in ethylene glycol. JavedIjajHussain et al. in the year 2011 [8] reported chemical reduction process to synthesize stable and various shapes of silver nanoparticles in water by the use of reducing agents. Jie et al. [9] in the year 2008 reported the thermal reduction method for synthesis of Ag nanoparticles. Poonam Pal Singh et al. [10] in the year 2012 reported the green synthesis of $\mathrm{Ag}$ nanoparticles from leaves and barks. The biosynthesis of nanoparticles as an emerging highlight of the intersection of nanotechnology and biotechnology has increased the attention due to a growing need to develop environmentally benign technologies in material synthesis (Kalishwaralal et al. 2008). The first evidence of synthesizing silver nanoparticles by green route was established in 1984 using Pseudomonas stutzeri (Haefeli et al. 1984, Zhang et al. 2005; Nair and Pradeep 2002). Huang et al. in the year 2008 suggested the use of Cinnamomumcamphora leaves to synthesise the Ag nanoparticles. Various works have been reported where Ag nanoparticles are synthesized by the use of templates like DNA, membrane, viruses and diatoms [11]. 


\section{Experimental Section}

\subsection{Synthesis}

Silver nitrate $\left(\mathrm{AgNO}_{3}\right)$ (assay $\geq 99 \%$ ) was purchased from Merck Specialities Private Limited, fresh tea leaves were collected from tea garden of upper Assam. The Ag nanoparticles (figure 2) are synthesized via green route (12) taking tea leaf extract (figure 2) which serves both as capping agent and stabilizer.

All the as-prepared samples are then subjected to various characterization techniques.

Table 1. Physical parameters for Ag/tea extract samples.

\begin{tabular}{|c|c|c|c|c|}
\hline Sample Code & Volume of $\mathrm{AgNO}_{3}(\mathrm{ml})$ & Volume of Tea extract (ml) & Volume of water $(\mathrm{ml})$ & Total Volume (ml) \\
\hline $\mathrm{A}_{\mathrm{g}} \mathrm{G}_{1}$ & 0.2 & 0.01 & 3.79 & 4 \\
\hline $\mathrm{A}_{\mathrm{g}} \mathrm{G}_{2}$ & 0.2 & 0.05 & 3.75 & 4 \\
\hline
\end{tabular}

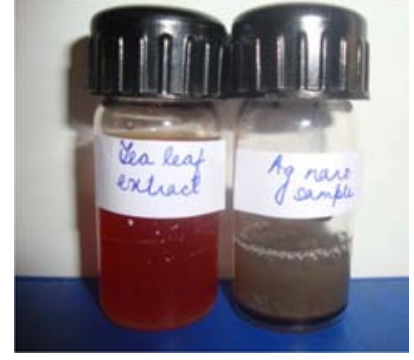

Figure 2. Tea leaf extract and the Ag nano sample.

\subsection{Characterisation with Result Analysis}

\subsubsection{Optical Absorption Spectroscopy}

The UV-vis absorption spectra for the Ag nano samples $\mathrm{AgG}_{1}, \mathrm{AgG}_{2}$ silver nitrate solution \& tea leaves extract are shown in figures $3(\mathrm{a}, \mathrm{b})$ and 4 respectively. The SPR wavelength of the as-fabricated samples with the size determined from equation 1 is given in table 2 .

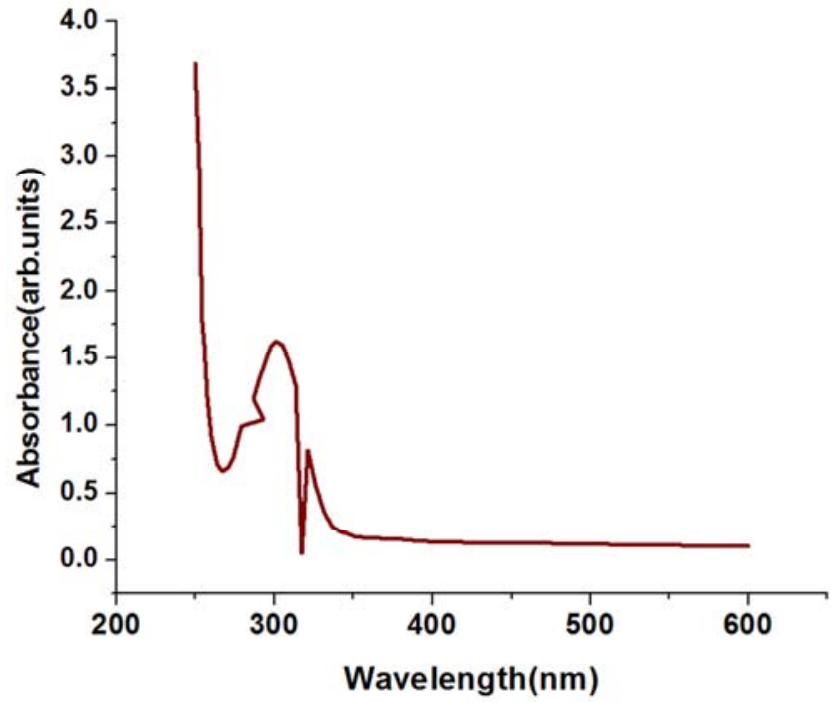

(a)

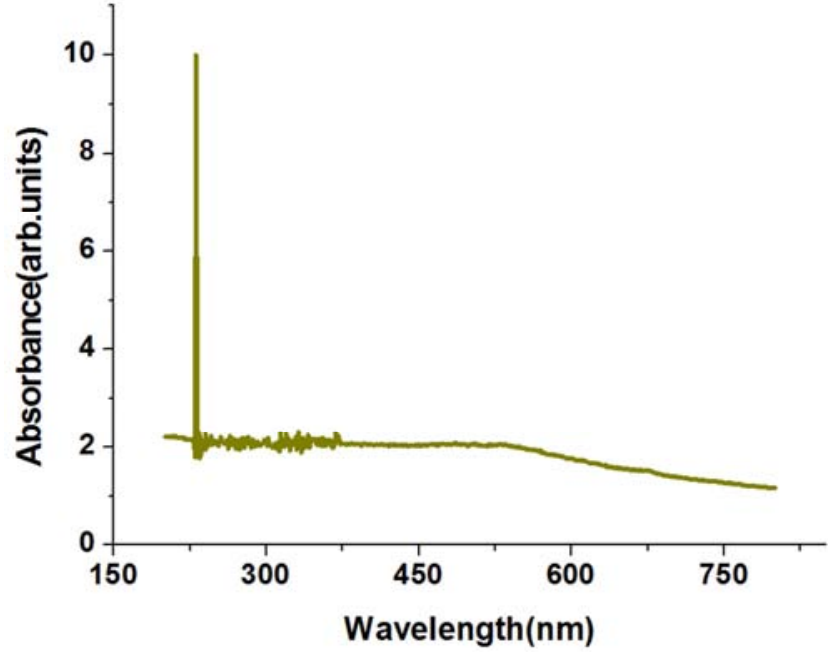

(b)

Figure 3. UV-Vis spectra of (a) Silver nitrate solution, (b) tea leaf extract.

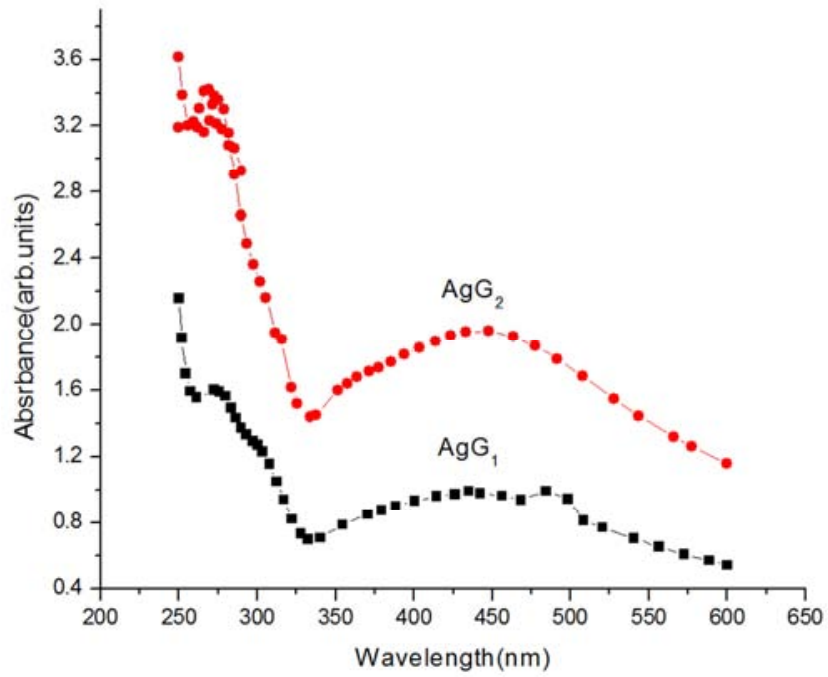

Figure 4. UV-vis spectra of the Ag nano samples $\mathrm{AgG}_{1}$ and $\mathrm{AgG}_{2}$.

Table 2. Surface Plasmon resonance peak and the size estimated for the as-fabricated Ag nano samples.

\begin{tabular}{lll}
\hline Sample Code & Wavelength corresponding to the SPR peak (nm) & Size (nm) \\
\hline $\mathrm{AgG}_{1}$ & $438.8,447.4$ & 28.56 \\
$\mathrm{AgG}_{2}$ & 437.2 & 28.30 \\
\hline
\end{tabular}

The optical absorption spectra of the silver nanoparticles are dominated by Localised Surface Plasmon Resonances
(LSPR). The LSPR peaks for the as-fabricated samples of Ag nanoparticles are provided in table 2. Moreover, in the 
sample $\mathrm{AgG}_{2}$, the presence of two distinct peaks are observed, one at $438.8 \mathrm{~nm}$ and the other at $447.4 \mathrm{~nm}$. The position and the shape of the absorption spectra depends on particle size and shape, the stabilizer used in the synthesis of the nanoparticles. According to Mie theory, only a single LSPR peak is expected in the absorption spectra of isotropic silver nanoparticles whereas two or more peaks are observed in anisotropic particles [13]. It is found that the number of SPR peaks increases as the symmetry of the nanoparticles decreases. Thus spherical np show one peak and disks, triangular or asymmetric nps show two or more peaks.
Yuning Li et al [14] in their experiment of fabrication of high conductivity elements for printed electronic found the existence of surface Plasmon peak for $\mathrm{Ag} \mathrm{np}$ at $415 \mathrm{~nm}$. Similar results are also obtained by other workers $[13,14$, $15]$.

\subsubsection{Photoluminescence (PL) Spectroscopy}

The photoluminescence spectra obtained for the samples $\mathrm{AgG}_{1}, \mathrm{AgG}_{2}, \mathrm{AgG}_{3}$ and $\mathrm{AgG}_{4}$ are as shown in figure 5. The PL peaks for all the samples lies within 745 to $750 \mathrm{~nm}$.

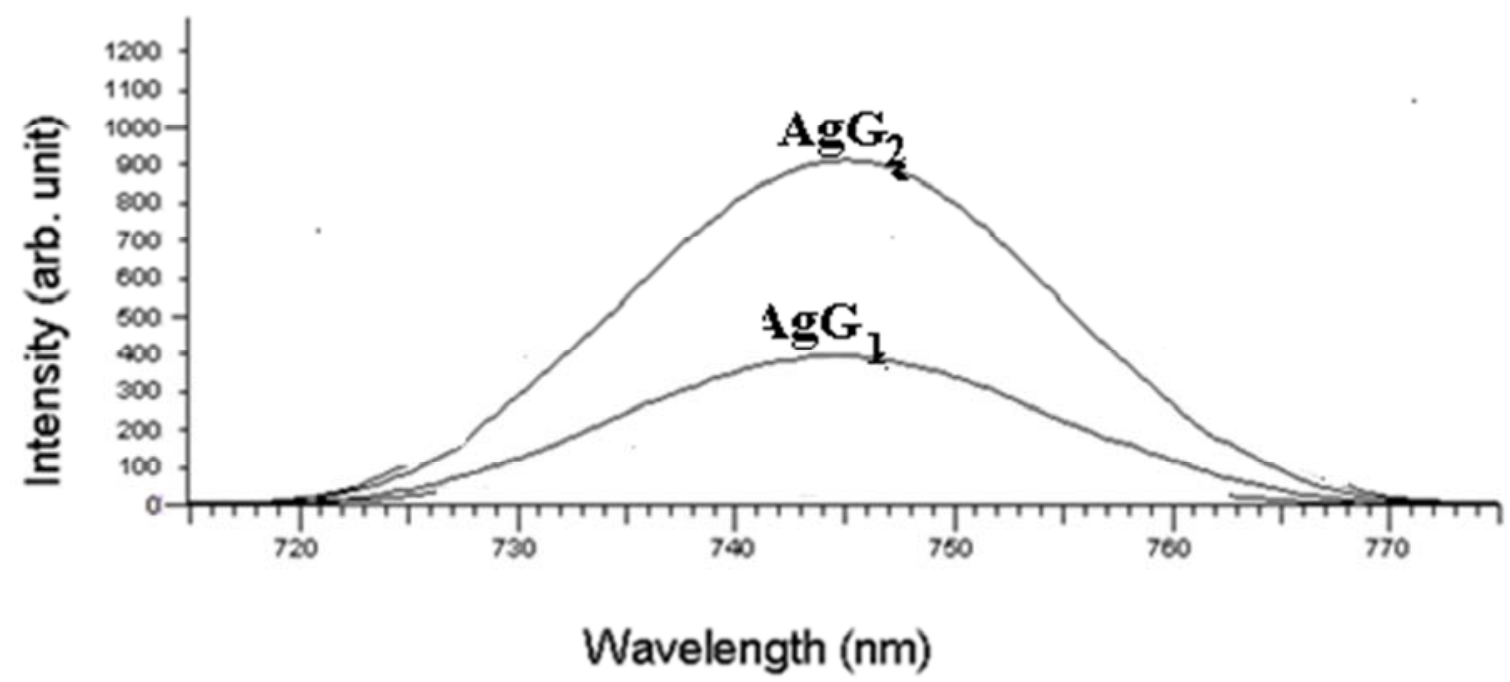

Figure 5. $P L$ spectra of the samples $A g G_{1}, A g G_{2}$.

The visible luminescence of Ag nanoparticles is due to the excitation of the electrons from the occupied ' $d$ ' bands into states above the Fermi level. Subsequent relaxation by the electron-phonon scattering process leads to an energy loss and finally the photoluminescent radiative recombination of an electron from an occupied sp band with hole takes place. The optical properties of Ag nanoparticles depend on both the interband and intraband transitions between electronic states.

With $\mathrm{Ag}$ nps are synthesized by using $\mathrm{AgNO}_{3}$, gelatin, glucose and $\mathrm{NaOH}$, Ramakrishna Vasireddy et al [16] observed PL emission within the visible range, from 400 to $600 \mathrm{~nm}$. Similar results are also obtained by other workers [17].

\subsubsection{High Resolution Transmission Electron Microscopy (HRTEM) and Selected Area Electron Diffraction (SAED)}

Figures 6 and 7 depict the HRTEM images of the sample $\mathrm{AgG}_{1}$ and $\mathrm{AgG}_{2}$ respectively. The morphology of the nanoparticles is depicted from these pictures. Figure 6 shows that the shape of the nanoparticles formed for the sample $\mathrm{AgG}_{1}$ is non uniform with two axes of symmetry and the nanoparticles of different shapes are formed. Thus, the inferences drawn from UV-vis pattern (figure 4) are supported by the HRTEM pictures (figures 6\&7). The figures 6(e) \& 7(d) shows the SAED picture. The d-spacing obtained is of the value $0.23 \mathrm{~nm}$. The formation of a single peak in the sample $\mathrm{AgG}_{2}$ in the UV-Vis spectrum is also revealed from the HRTEM images of the said sample. The images shown in figure 6 show that most of the particles are spherical in shape with a smooth surface morphology with an average diameter of $\sim 20 \mathrm{~nm}$. It is also seen that few particles are present whose diameter is less than $10 \mathrm{~nm}$. The picture clearly shows the lattice planes of the crystal with d-spacing of $0.20 \mathrm{~nm}$.

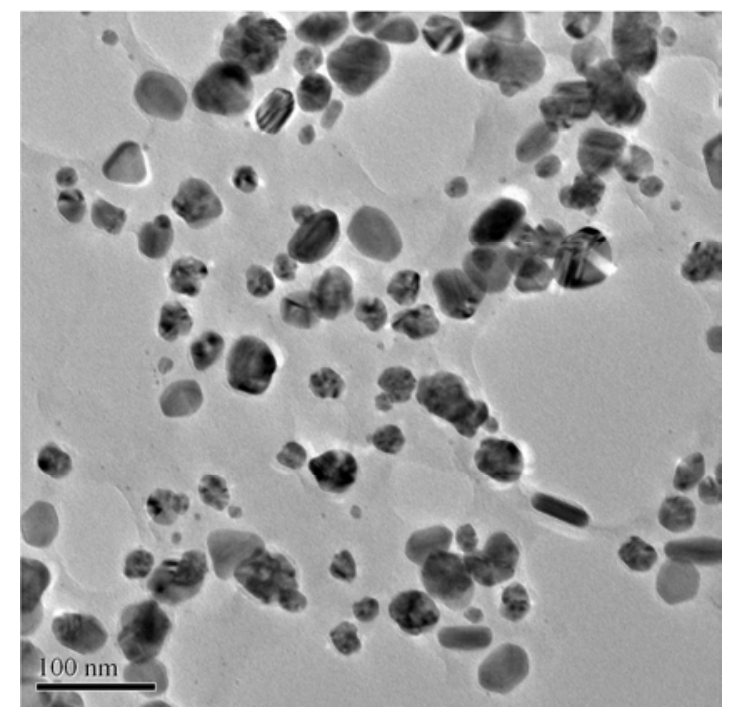

(a) 


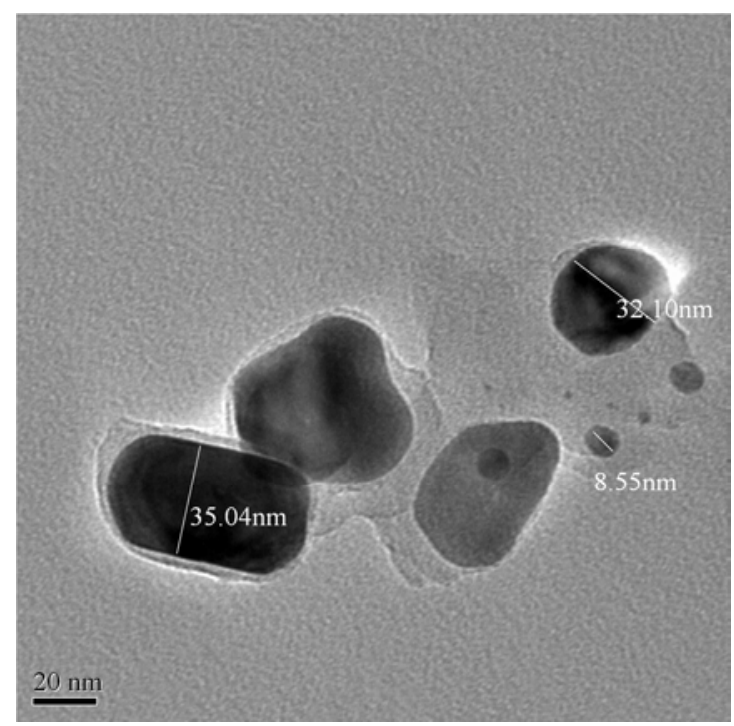

(b)

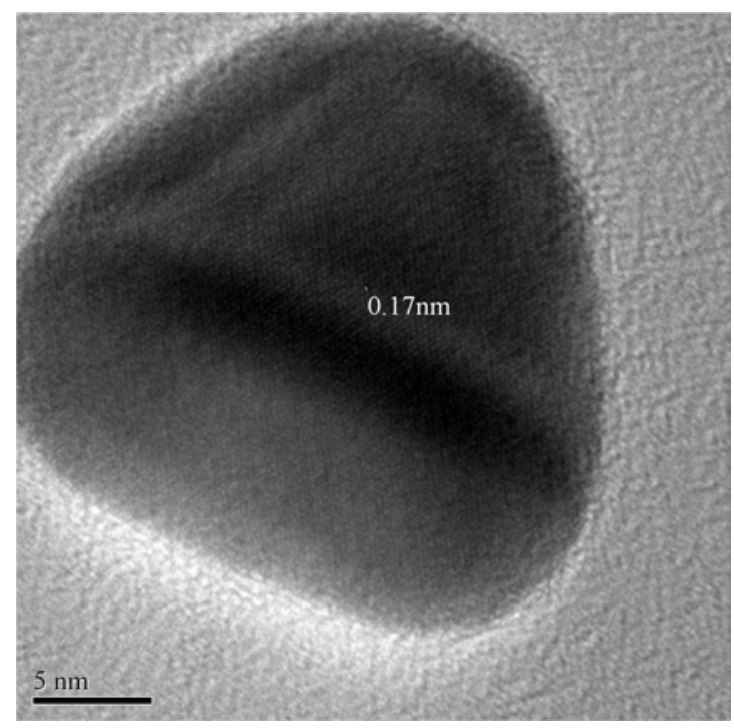

(c)

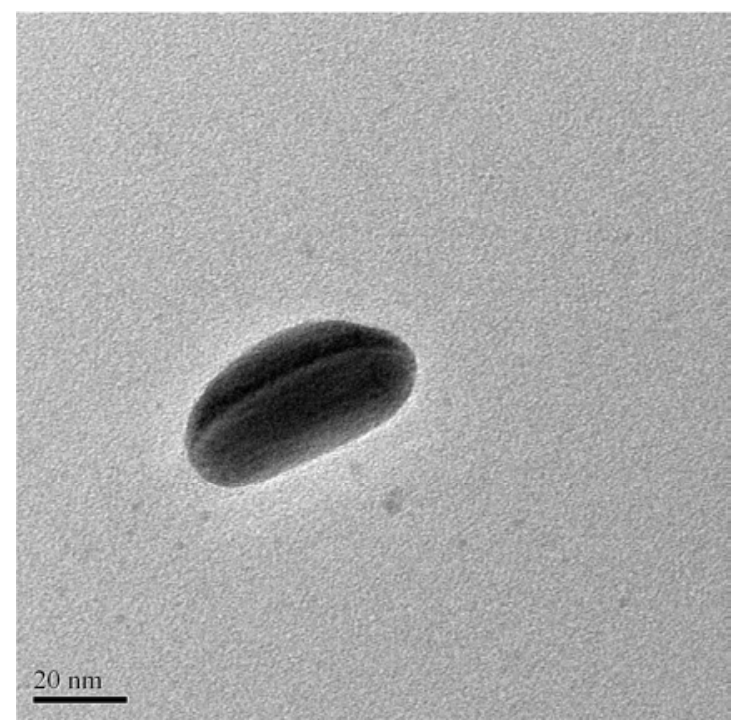

(d)

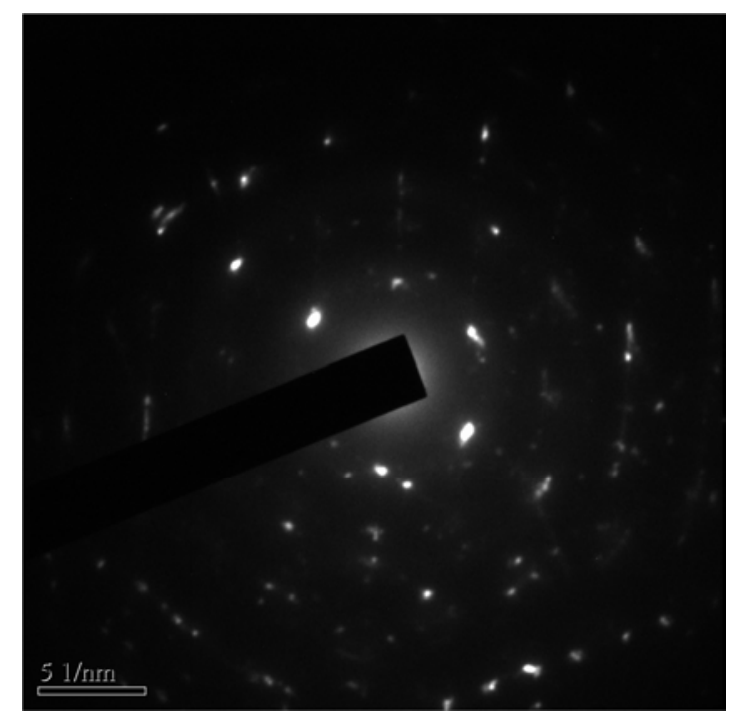

(e)

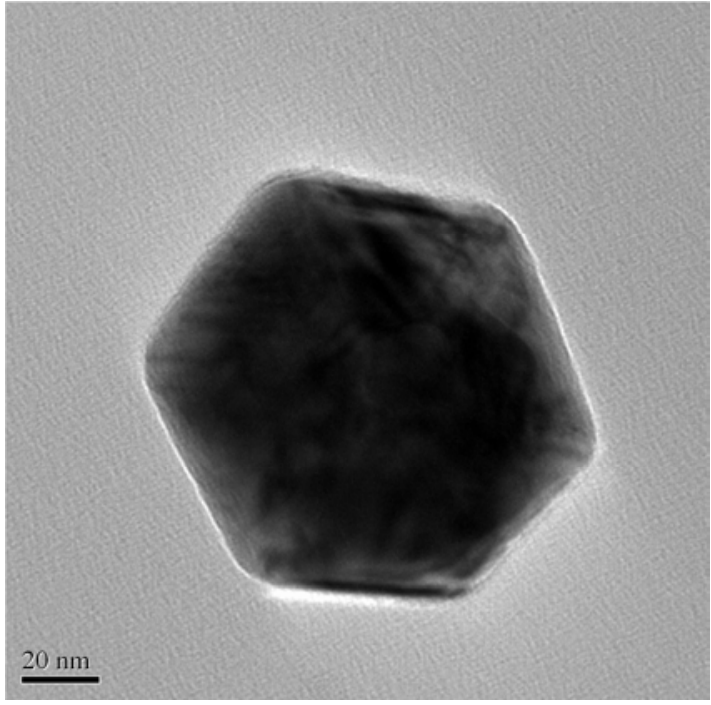

(f)

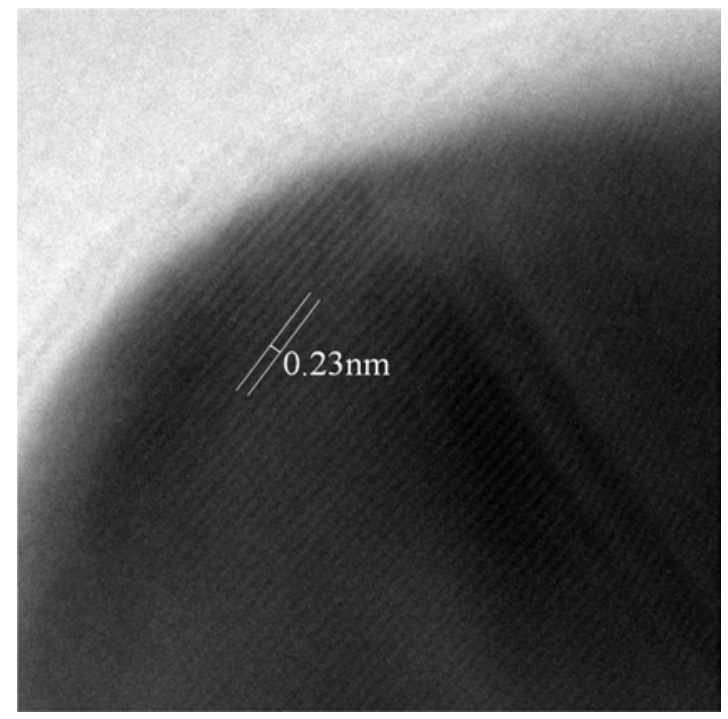

(g)

Figure 6. $(a),(b),(c),(d),(e),(f)$ and $(g)$ HRTEM pictures of the sample $A g G_{I}$. 


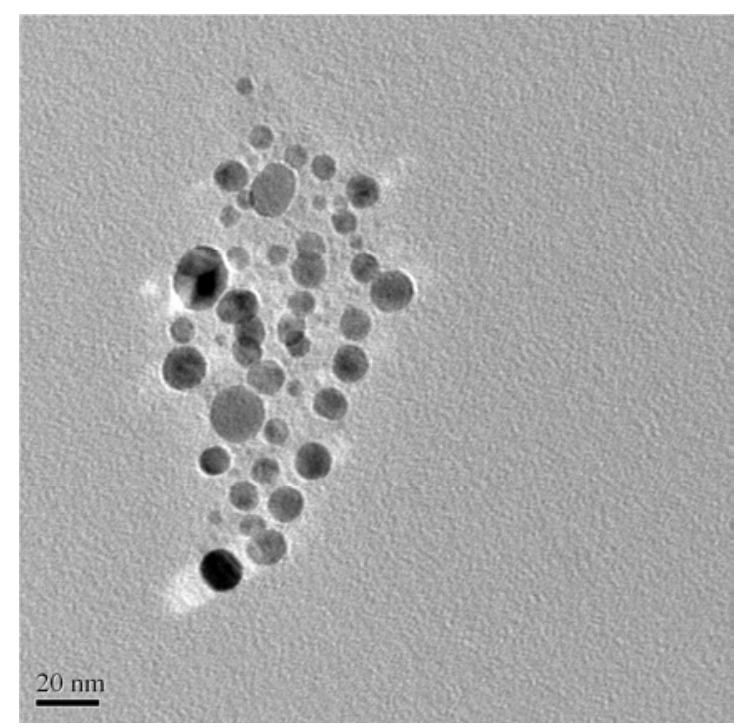

(a)

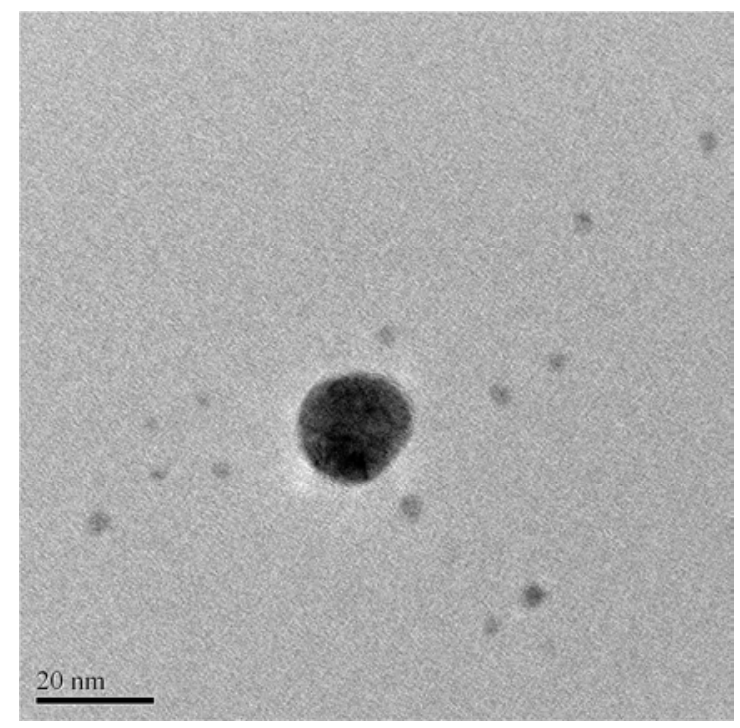

(b)

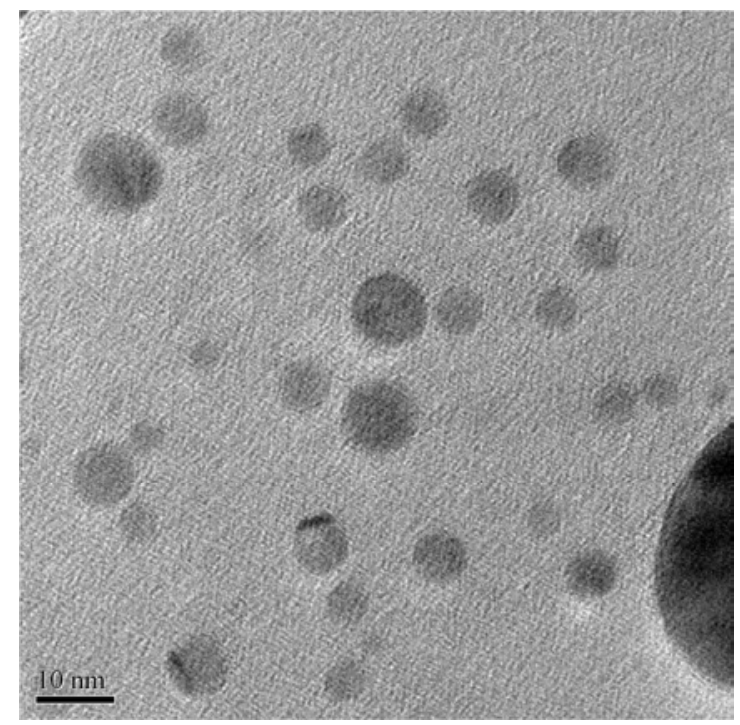

(c)

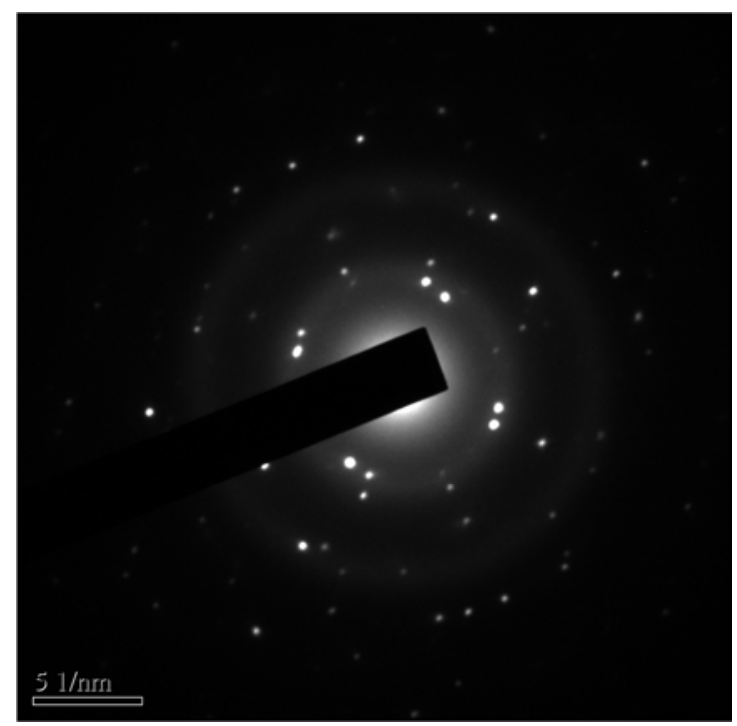

(d)

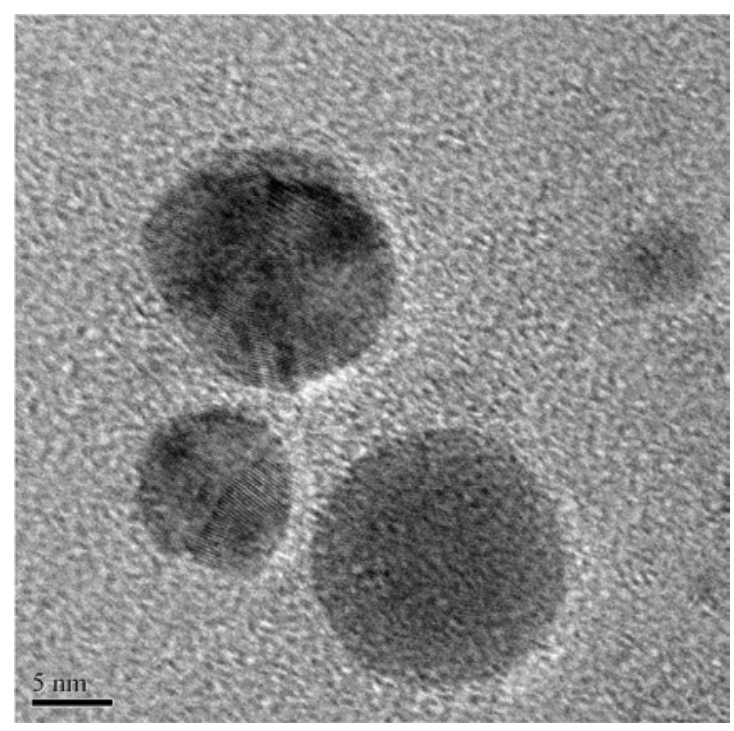

(e)

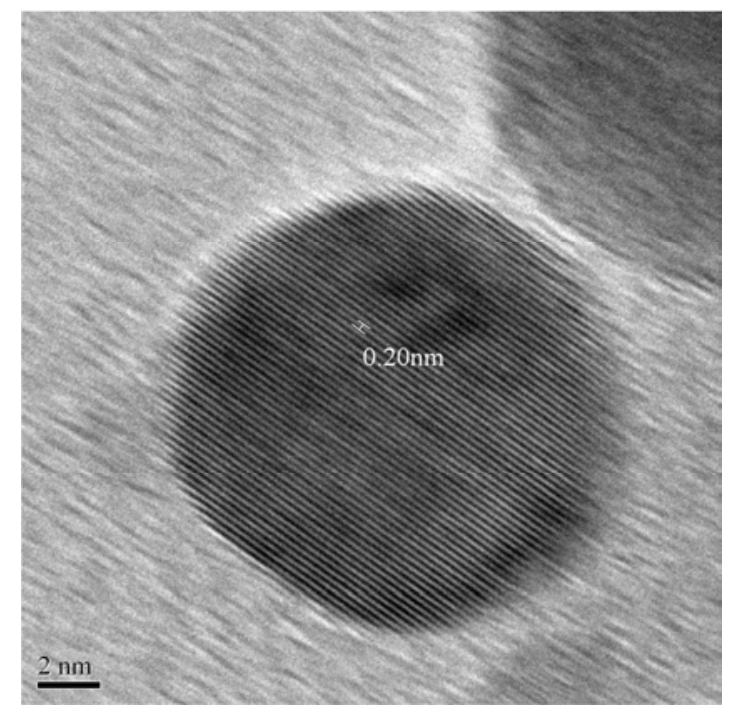

(f)

Figure 7. (a),(b), (c),(d),(e) and (f) HRTEM images of the sample $A g G_{2}$. 
Table 3 is the composite table giving the size obtained from various characterisation techniques for the as fabricated
Ag particles in tea leaf extract

Table 3. Size estimated for Ag/tea extract samples from various characterisation techniques.

\begin{tabular}{|c|c|c|c|c|c|c|c|}
\hline \multirow{2}{*}{ S. No } & \multirow{2}{*}{ Sample Code } & \multirow{2}{*}{ Volume of $\mathrm{AgNO}_{3}(\mathrm{ml})$} & \multirow{2}{*}{ Volume of Tea extract (ml) } & \multirow{2}{*}{ Volume of water $(\mathrm{ml})$} & \multirow{2}{*}{ Total Volume (ml) } & \multicolumn{2}{|c|}{ Average diameter } \\
\hline & & & & & & UV-Vis & HR-TEM \\
\hline 1 & $\mathrm{AgG}_{1}$ & 0.2 & 0.01 & 3.79 & 4 & 28.30 & 30 \\
\hline 2 & $\mathrm{AgG}_{2}$ & 0.2 & 0.1 & 3.7 & 4 & 27.64 & 20 \\
\hline
\end{tabular}

\section{Conclusion}

In the present work, Ag nanoparticles are fabricated by green route taking tea leaf extract which acts as both stabilizer and capping agent. For fabrication of $\mathrm{Ag}$ nps by green route, the volume of the $\mathrm{AgNO}_{3}$ solution, volume of tea leaf extract and the volume of water are taken as the variable parameters. UV-vis characterisation results for the Ag samples, SPR peak is found to be present in the proper wavelength range. Size estimated for $\mathrm{Ag} /$ tea extract nps from the SPR peaks are in the range (18-34) $\mathrm{nm}$. Though the photoluminescence is rare in metals due to the lack of band gap, we have observed PL peaks for the as-synthesised Ag nps. The observed luminescence peaks can be attributed to the excitation of the electrons from the occupied ' $d$ ' bands into states above the Fermi level. It is found from the HRTEM pictures, for $\mathrm{Ag} /$ tea extract samples, clear pictures with no agglomeration. Also, it is seen that almost all Ag/tea extract particles are spherical in shape with size ranging from $(15-30) \mathrm{nm}$. For the sample $\mathrm{AgG}_{2}$ the shape of the particles are found to be non-spherical, which is also supported by the corresponding result of UV-Vis having two peaks. The SAED results confirm the value of d-spacing for the asfabricated Ag nano samples. Sizes of particles (15-30) nm are found to be much larger than the Fermi wavelength of Ag. Thus, it can be concluded that in the present investigation, Ag nanoparticles embedded tea extract are successfully synthesized. The shapes of the as synthesized nanoparticles are almost uniform in the sample with one peak in the UVVis spectrum whereas the different shaped particles can be seen in the sample with two peaks in the spectrum.

\section{Acknowledgements}

1) Optical absorption Spectroscopy: The optical absorption studies are made on HITACHI U-3210 double beam spectrophotometer available in the Dept. of Chemistry, G. U, Assam.

2) Photoluminescence Spectroscopy: The photoluminescence is recorded with HITACHI F-2500 fluorescence spectrophotometer available in the Dept. of Chemistry, G. U, Assam.

3) High Resolution Transmission Electron Microscopy and Electron Diffraction: High resolution transmission electron microscopy as well as electron diffraction is performed on JEOLJEM2100 microscope (at $200 \mathrm{KV}$ with point resolution of $1.44 \AA$, line resolution of $2.32 \AA$ and having super twin lenses) available at SAIF, North East Hill University (NEHU), Shillong, Meghalaya.

\section{References}

[1] Zeng, S.; Yong, Ken-Tye; Roy, Indrajit, Dinh, Xuan-Quyen; Yu, Xia; Luan, Feng. (2011) Plasmonics 6(3):491-506.

[2] Rich, RL; Myszka, DG, (2007). Anlytical biochemistry 361(1): $1-6$.

[3] Dr Ashutosh Sharma, Dr Jayesh Bellare, Dr Archana Sharma. (2006)Advances in Nanoscience \& Nanotechnology. National Institute of Science Communication and Information Resources.

[4] Jeremy R Dunklin and D. Keith Roper. (2017) Hindawi, Journal of Nanomaterials, 1D 2753934.

[5] C. Petit, P. Lixon, and M. P. Pileni, (1990). J. Phys. Chem. 94, 1598.

[6] Poonam Benjwal, thesis on single pot synthesis of bio compatible silver nanocolloids. [2010]. Availableon http://dspace.thapar.edu:8080/dspace/bitstream/10266/1161/3/ 1161.pdf

[7] S. Navaladian, B Viswanathan, R. p viswanath, T. K. Varadarajan, (2007) Nanoscale Res. Lett. 2:44-48.

[8] Javed Ijaj Hussain, Sunil Kumar, Athar Adil Hashmi, Zaheer Khan, (2011) Adv. Mat. Lett, 2(3), 188-194.

[9] Jie Zheng, Yong Ding, Bozhi Tian, Zhong Lin Wang, and Xiaowei zhuang, (2008) J. Am. Chem. Soc., 130, 10472-10473.

[10] Prasoon Pal Singh, Chittaranjan Bhakat, International Journal of Scientific andResearch Publications, (2012) Volume 2, Issue 5.

[11] Ramakrishna Vasireddy, Rima Paul and Apurba Krishna Mitra. (2012). Nanomaterials and Nanotechnology.

[12] Lipi Goswami, Debabrata Baishya, Sorra Sandhya, Joyeeta Talukdar, (2014), 5(2) International Journal of Pharma and Biosciences, 126-204.

[13] Z. Khan, J. Hussain, S. Kumar, A. Hashmi and M. Malik, (2011), 2 (4), Journal of Biomaterials and Nanobiotechnology, 390-399.

[14] Yuning Li, Yiliang Wu, and Berg. Ong. (2005), 127 (10), J. Am. Chem. Soc. 3266-3267.

[15] Asmita J. Gavhane, P. Padmanabhav, Suresh P. Kamble and Suresh N. Jangle.(2012), 3(3) Int J Pharm Bio Sci, 88-100.

[16] Ramakrishna Vasireddy, Rima Paul and Apurba Krishna Mitra. (2012), 2(8) Nanomaterials and Nanotechnology.

[17] Y. B Wankhede, S. B Kondawar, S. R. Thakare, P. S More. (2013), 4(1), Advanced Materials Letters, 89-93. 